\title{
Arbeitskreis Psychosomatische Dermatologie (APD) Sektion der DDG
}

\author{
"Der alte Haut-Patient" - 11. Jahrestagung Gießen \\ (31.01 - 01.02.2003)
}

Freitag, den 31.01.2003

12.00 Uhr Vorstandssitzung APD

14.00 Uhr Begrïßung und Eröffnung der Tagung

Höring - Vorsitzende des APD

Jecht - Berufsverband der Dermatologen

Schulz - Dekan des Fachbereichs

Humanmedizin

Eichert -Ehrenvorsitzende des APD

Gieler - Tagungsleiter

14.30 Uhr Compliance-Probleme bei alten Hautpatienten Taube / Halle

15.00 Uhr Freie Vorträge - Wissenschaftliches Programm

15.30 Uhr

16.00 Uhr

16.30 Uhr

17.00 Uhr

17.30 Uhr

Pause

Wenn Psychotherapeuten älter werden - Wie fühlt sich der Psychotherapeut mit 30, 40, 50, 60,70 und 80 ?

Eichert / Koblenz

18.00 Uhr Mitgliederversammlung und Neuwahl des

Vorstandes

20.00 Uhr Come-Together-Party im Gate 5

ohne Vortrag, aber mit Musik

Samstag, den 01.02.2003

9.00 Uhr Dia - Klinik (4 Fälle aus der psychosomatischen Dermatologie zum Thema)

10.00 Uhr Der alte Patient - Psychosomatische Aspekte Peters / Bad Berleburg
10.30 Uhr Der alte Patient - Dermatologische Aspekte Effendy / Bielefeld

11.00 Uhr Pause

11.30 Uhr Seminare zum Thema: «Der alte Patient»

13.00 Uhr Mittagspause

14.00 Uhr Fall-Demonstration durch Video und Diskussion aus unterschiedlichen psychotherapeutischen Schulen

15.30 Uhr Ende der Tagung

16.00 Uhr Vorstandssitzung APD

Seminarthemen zur Weiterbildung psychosomatische Grundversorgung in der Dermatologie (wird zertifiziert!)

Seminar 1: Bedeutung des Erstkontaktes in der psychosomatisch-dermatologischen Sprechstunde

(Leitung: Höring / Stuttgart)

Seminar 2: Der alte Patient in der Psychotherapie (Leitung: Peters / Bad Berleburg)

Seminar 3: Der alte Patient in der Dermatologie (Leitung: Effendy / Bielefeld)

Seminar 4: Artefakte, Simulationen, Parartefakte - Erkennen und Behandeln (Leitung: Gieler / Gießen)

Seminar 5: Die Neurodermitis-Schulung, Einführung (Leitung: Hennighausen / Gießen)

Seminar 6: Körpertherapie bei Hautkrankheiten (Leitung: Schreiber / Gießen)

Tagungsorganisation und weitere Informationen:

Prof. Dr. U. Gieler

Zentrum für Psychosomatische Medizin

Ludwigstraße 76, D-35392 Gießen

Tel. +49 $64199-45650$ oder 45651

Fax +49641 99-45659

E-mail Uwe.Gieler@psycho.med.uni-giessen.de

Homepage: www.med.uni-giessen.de/psychoderm/html 